\title{
Mitochondrial DNA heteroplasmy in human health and disease (Review)
}

\author{
GEORGE B. STEFANO and RICHARD M. KREAM \\ MitoGenetics, LLC, Farmingdale, NY 11735, USA \\ Received December 18, 2015; Accepted February 2, 2016
}

DOI: $10.3892 /$ br. 2016.590

\begin{abstract}
The biomedical literature has extensively documented the functional roles of genetic polymorphisms in concert with well-characterized somatic mutations in the etiology and progression of major metastatic diseases afflicting human populations. Mitochondrial heteroplasmy exists as a dynamically determined co-expression of inherited polymorphisms and somatic mutations in varying ratios within individual mitochondrial DNA genomes with repetitive patterns of tissue specificity. Mechanistically, carcinogenic cellular processes include profound alterations of normative mitochondrial function, notably dependence on aerobic and anaerobic glycolysis, and aberrant production and release of lactate, according to a classic theory. Within the translational context of human health and disease, the present review discusses the necessity of establishing critical foci designed to probe multiple biological roles of mitochondrial heteroplasmy in cancer biology.
\end{abstract}

\section{Contents}

1. Introduction

2. Biological significance of mitochondrial heteroplasmy

3. Mitochondrial heteroplasmy and cancer

4. Conclusions

\section{Introduction}

Extensive biomedical literature has documented the functional roles of heritable genetic polymorphisms in concert with accumulated somatic mutations and epigenetic factors in the

Correspondence to: Dr George B. Stefano, MitoGenetics, LLC, Suite 307, 3 Bioscience Park Drive, Farmingdale, NY 11735, USA

E-mail: george.stefano@mitogenetics.com

Abbreviations: mtDNA, mitochondrial DNA; WT, wild-type; CO1, cytochrome $c$ oxidase subunit I; iPSCs, induced pluripotent stem cells

Key words: mitochondria, mitochondrial DNA, mitochondrial DNA heteroplasmy, bioenergetics, somatic mutation etiology and progression of major metastatic $(1,2)$, autoimmune $(3,4)$ and neurodegenerative $(2,5,6)$ disorders. By contrast, a considerably smaller subset of heritable polymorphisms in the haploid mitochondrial DNA (mtDNA) genome is functionally linked to the phenotypic manifestation of severe genetic disorders originating from markedly compromised mitochondrial function (7-10). Notably, a relatively recent body of literature has demonstrated interactive effects of heritable mtDNA polymorphisms on the altered expression of select nuclear genes that are associated with several human disease states (11-13). Conversely, specific allele imbalances within the nuclear mitochondrial genome are associated with altered translocation of key mitochondrial proteins involved in normative bioenergetics and are negatively linked to cancer progression $(14,15)$.

\section{Biological significance of mitochondrial heteroplasmy}

Mitochondrial heteroplasmy may be defined as a dynamically determined co-expression of wild-type (WT)-inherited polymorphisms and somatic mutations in varying ratios within individual mtDNA genomes distributed throughout the intraorganelle compartments of individual cells. Within the translational context of human health and disease, the necessity of establishing critical foci designed to probe multiple biological roles of mitochondrial heteroplasmy has become apparent. Human mtDNA exists as a 16.6-kilobase circular genome that contains 13 protein-encoding sequences corresponding to subunits ND1-6, including ND4 and ND4L, of respiratory complex I, catalytic subunits cytochrome $c$ oxidase subunit I-III (CO1-3) of respiratory complex IV, subunits adenosine triphosphate 6 (ATP6) and ATP8 of F1F0 ATPase, and cytochrome B of respiratory complex III. The remaining genes encode 22 tRNAs and 12, and 16S rRNAs (16). As the number of mitochondrial genomes normalized against each diploid nuclear genome varies according to cell type and total mtDNA copy number, estimated values of 100-10,000 have been reported in the biomedical literature $(8,16,17)$. The complex sequence heterogeneity within mixed intra-mitochondrial populations of several thousand individual heteroplasmic mtDNA genomes within individual cells dictates that empirical sorting of putative biological activities of distinct patterns of mtDNA heteroplasmy, according to molecular biological, biochemical, physiological and bioinformatic criteria will be technically difficult (18-20).

Notably, a previous study monitored the intra-mitochondrial organization of heterologous heteroplasmic mtDNA 
genomes into DNA-protein complexes termed nucleoids (21). Heterologous mtDNAs were stably maintained in distinct nucleoid populations, whereas trans-complementation of heteroplasmic nucleoids was apparently achieved by the diffusion of mtDNA-derived transcripts within the mitochondrial matrix. Although the investigators speculated on a putative restorative mechanism of trans-complementation to operationally increase homoplasmic WT mtDNA and mitochondrial bioenergetics, other studies have demonstrated distinct and repeatable patterns of mtDNA heteroplasmy that varied across different cell types from the same individual $(22,23)$ and were stably maintained in individual daughter cells over multiple cell divisions (19). An ostensibly straightforward interpretation of these observations indicates highly regulated normative expression of heteroplasmic mtDNA genomes within the intra-mitochondrial compartment in individual human cell types that complements normative mitochondrial function. Mechanistically, tissue- and cell-specific patterns of heteroplasmic mtDNA appear to be maintained via intra-mitochondrial trans-complementation of heteroplasmic nucleoids and mtDNA-derived transcripts (21), as well as the intercellular exchange of mtDNA (19). It also becomes apparent that the polycistronic nature of heteroplasmic mtDNA-encoded transcripts introduces an additional level of complexity by which to evaluate putative facilitative roles of preserved patterns of mtDNA heteroplasmy on homeostatic metabolic processes (16).

Validation of the potential existential role of cell-specific patterns of mtDNA heteroplasmy on normative mitochondrial functions is also provided by a preclinical Drosophila genetic model employing a temperature sensitive-lethal mtDNA mutation in functional linkage to the CO1 locus (24). Notably, the viability of homoplasmic flies at restrictive temperatures was fully maintained by expressing an alternative CO1 oxidase, which specifically conferred restorative mtDNA heteroplasmy that was associated with fully viable and tissue-specific phenotypes. Conversely, using a genetic replacement paradigm to induce tissue-specific mutant $\mathrm{CO} 1$ homoplasmy in heteroplasmic flies, it was observed that restoration of mtDNA homoplasmy in the eye resulted in severe neurodegeneration at restrictive temperatures. Of note, utilizing the same temperature sensitive-lethal mtDNA model of CO1 dysfunction, the frequency of the mutant allele in heteroplasmic flies was significantly decreased in the germline and over multiple generations (25). A critical analysis of these two studies concludes that selection against potentially deleterious mtDNA heteroplasmic mutations during the process of oogenesis may be in marked contrast to developmentally determined, cell-specific patterns of mtDNA heteroplasmy generated during various stages of tissue differentiation.

\section{Mitochondrial heteroplasmy and cancer}

The mechanistic staging of carcinogenic cellular processes includes profound alterations of normative mitochondrial function (26-29), notably dependence on aerobic and anaerobic glycolysis, aberrant production and release of lactate, and metabolic downregulation of mitochondrial oxidative processes according to the classic theory promoted by Warburg et al (30). The primacy of cancer as a mitochondrial metabolic disease has been proposed (31-33), in marked contrast to widely espoused theories supporting the causative role of multiple somatic mutations in the etiology and persistence of numerous types of cancer. In light of the relatively high somatic mutational rates of mtDNA, the multi-modal coding mechanisms provided by cell-specific patterns of mtDNA heteroplasmy may reflect the evolutionary linkages of mitochondria to primordial protobacterial precursors and trillions of enteric bacteria contained within the human microbiome (20,34-36). As a corollary, cellular processes that regulate physiologically compatible patterns of heteroplasmic mtDNA may undergo state-dependent dysregulation resulting in an altered metabolically compromised phenotype characteristic of cancer cells and other pathophysiologically altered cell types $(22,37)$. A relevant previous study has linked allele-specific expression of nuclear DNA with mtDNA heteroplasmy with functional impairment in bioenergetics and an apparent positive selection for reduced mitochondrial function (38). The overall results and conclusions of the study provide supportive evidence for positive selection processes driving higher order cellular pattern recognition of heteroplasmic mtDNA genomes in ordered stages of tumor progression $(18,22,23,39)$.

Recently, a critical review has outlined a binary regulatory system responsible for selective expression of genes contained within mitochondrial and chloroplast genomes (40). The evolutionarily conserved mtDNA genome represents a self-contained genetic system that encodes existentially required catalytic and regulatory subunits of respiratory complexes I, III and IV, and two subunits of F1F0 ATPase. The unifying principle responsible for reciprocal regulation of intraorganelle energy production is critically linked to maintenance of redox potential by electron transport through respiratory complexes. Accordingly, the functional transformation of cell-specific mitochondria into high-efficiency bio-engines appears to be dependent on the veracity of ongoing gene expression within restricted metabolic or physiological demands. The critical regulatory roles of cell-specific patterns of mtDNA heteroplasmy are fundamental to the maintenance of requisite metabolic capacity during the normal aging processes (18-20,39). These contentions are also supported by a recent study that has underlined the critical importance of functional mitochondria in the maintenance of differentiation and reprogramming of induced pluripotent stem cells (iPSCs) (41). Notably, a transition from somatic mitochondrial oxidative metabolism to glycolytic metabolism, highly reminiscent of cancer cells, was observed to be required for successful reprogramming of iPSCs. Accordingly, somatic mitochondria and associated oxidative bioenergetics are extensively remodeled with the induction of an iPSC-like phenotype, and the transition from oxidative to glycolytic metabolic processes appears to be strongly regulated by hypoxia, specifically by hypoxia-inducible factor $1 \alpha(\mathrm{HIF} 1 \alpha)$ signaling pathways $(42,43)$. Early induction of HIF1 $\alpha$ target genes may be required for iPSC derivation via the activation of a glycolytic program that is highly reminiscent of undifferentiated cancer cells.

\section{Conclusions}

We hypothesize that the biological significance of mtDNA heteroplasmy is reflected by the ability of cellular mitochondria to modulate effectively state-dependent changes in energy 
requirements by concerted transcriptional and translational mechanisms $(19,44,45)$. An apparent perturbation of homeostatic regulation of intra-mitochondrial patterns of mtDNA heteroplasmy may be amplified during the initiation and progression of pathophysiological processes associated with major human disease states $(17,19,23,46,47)$. Furthermore, bidirectional communication between cytosolic and mitochondrial signaling pathways provide co-ordinate regulation of nuclear DNA- and mtDNA-derived gene expression within a constantly changing physiological environment designed to promote molecular switching of cellular metabolic machinery from meeting anabolic to catabolic demands (48). As technological transplantation of functionally viable mitochondria comes with the anticipation of the significant restoration of normative cellular function, understanding and mapping of cell-specific mosaic patterns of heteroplasmic mtDNA expression appears to represent a prime prerequisite for future translational studies.

\section{Acknowledgements}

The present study was funded by MitoGenetics, LLC (Sioux Falls, SD, USA).

\section{References}

1. Vogelstein B, Papadopoulos N, Velculescu VE, Zhou S, Diaz LA Jr and Kinzler KW: Cancer genome landscapes. Science 339: 1546-1558, 2013.

2. Frank SA: Somatic mosaicism and disease. Curr Biol 24: R577-R581, 2014.

3. Joseph CG, Darrah E, Shah AA, Skora AD, Casciola-Rosen LA, Wigley FM, Boin F, Fava A, Thoburn C, Kinde I, et al: Association of the autoimmune disease scleroderma with an immunologic response to cancer. Science 343: 152-157, 2014.

4. Ross KA: Coherent somatic mutation in autoimmune disease. PLoS One 9: e101093, 2014.

5. Poduri A, Evrony GD, Cai X and Walsh CA: Somatic mutation, genomic variation, and neurological disease. Science 341: 1237758,2013

6. Jamuar SS, Lam AT, Kircher M, D'Gama AM, Wang J, Barry BJ, Zhang X, Hill RS, Partlow JN, Rozzo A, et al: Somatic mutations in cerebral cortical malformations. N Engl J Med 371: 733-743, 2014.

7. Taylor RW and Turnbull DM: Mitochondrial DNA mutations in human disease. Nat Rev Genet 6: 389-402, 2005.

8. Song S, Pursell ZF, Copeland WC, Longley MJ, Kunkel TA and Mathews CK: DNA precursor asymmetries in mammalian tissue mitochondria and possible contribution to mutagenesis through reduced replication fidelity. Proc Natl Acad Sci USA 102 4990-4995, 2005.

9. Crimi M, O'Hearn SF, Wallace DC and Comi GP: Molecular research technologies in mitochondrial diseases: The microarray approach. IUBMB Life 57: 811-818, 2005.

10. Wallace DC: The mitochondrial genome in human adaptive radiation and disease: On the road to therapeutics and performance enhancement. Gene 354: 169-180, 2005.

11. Kenney MC, Chwa M, Atilano SR, Falatoonzadeh P, Ramirez C, Malik D, Tarek M, Cáceres-del-Carpio J, Nesburn AB, Boyer DS, et al: Inherited mitochondrial DNA variants can affect complement, inflammation and apoptosis pathways: Insights into mitochondrial-nuclear interactions. Hum Mol Genet 23 . 3537-3551, 2014

12. Kenney MC, Chwa M, Atilano SR, Falatoonzadeh P, Ramirez C, Malik D, Tarek M, Del Carpio JC, Nesburn AB, Boyer DS, et al: Molecular and bioenergetic differences between cells with African versus European inherited mitochondrial DNA haplogroups: Implications for population susceptibility to diseases. Biochim Biophys Acta 1842: 208-219, 2014.

13. Atilano SR, Malik D, Chwa M, et al: Mitochondrial DNA variants can mediate methylation status of inflammation, angiogenesis and signaling genes. Hum Mol Genet 24: 4491-4503, 2015.
14. Bera S, Weinberg F, Ekoue DN, Ansenberger-Fricano K, Mao M, Bonini MG and Diamond AM: Natural allelic variations in glutathione peroxidase-1 affect its subcellular localization and function. Cancer Res 74: 5118-5126, 2014.

15. Liu M, Li Y, Chen L, Chan TH, Song Y, Fu L, Zeng TT, Dai YD, Zhu YH, Li Y, et al: Allele-specific imbalance of oxidative stress-induced growth inhibitor 1 associates with progression of hepatocellular carcinoma. Gastroenterology 146: 1084-1096, 2014.

16. Anderson S, Bankier AT, Barrell BG, de Bruijn MH, Coulson AR, Drouin J, Eperon IC, Nierlich DP, Roe BA, Sanger F, et al: Sequence and organization of the human mitochondrial genome. Nature 290: 457-465, 1981.

17. Valero T: Mitochondrial biogenesis: Pharmacological approaches. Curr Pharm Des 20: 5507-5509, 2014.

18. Irwin JA, Saunier JL, Niederstätter H, Strouss KM, Sturk KA, Diegoli TM, Brandstätter A, Parson W and Parsons TJ: Investigation of heteroplasmy in the human mitochondrial DNA control region: A synthesis of observations from more than 5000 global population samples. J Mol Evol 68: 516-527, 2009.

19. Jayaprakash AD, Benson EK, Gone S, Liang R, Shim J, Lambertini L, Toloue MM, Wigler M, Aaronson SA and Sachidanandam R: Stable heteroplasmy at the single-cell level is facilitated by intercellular exchange of mtDNA. Nucleic Acids Res 43: 2177-2187, 2015.

20. Naue J, Hörer S, Sänger T, Strobl C, Hatzer-Grubwieser P, Parson W and Lutz-Bonengel S: Evidence for frequent and tissue-specific sequence heteroplasmy in human mitochondrial DNA. Mitochondrion 20: 82-94, 2015.

21. Schon EA and Gilkerson RW: Functional complementation of mitochondrial DNAs: Mobilizing mitochondrial genetics against dysfunction. Biochim Biophys Acta 1800: 245-249: 2010.

22. He Y, Wu J, Dressman DC, Iacobuzio-Donahue C, Markowitz SD, Velculescu VE, Diaz LA Jr, Kinzler KW, Vogelstein B and Papadopoulos N: Heteroplasmic mitochondrial DNA mutations in normal and tumour cells. Nature 464: 610-614, 2010.

23. Samuels DC, Li C, Li B, Song Z, Torstenson E, Boyd Clay H, Rokas A, Thornton-Wells TA, Moore JH, Hughes TM, et al: Recurrent tissue-specific mtDNA mutations are common in humans. PLoS Genet 9: e1003929, 2013.

24. Chen Z, Qi Y, French S, Zhang G, Covian Garcia R, Balaban R and $\mathrm{Xu} \mathrm{H}$ : Genetic mosaic analysis of a deleterious mitochondrial DNA mutation in Drosophila reveals novel aspects of mitochondrial regulation and function. Mol Biol Cell 26: 674-684, 2015.

25. Hill JH, Chen $\mathrm{Z}$ and $\mathrm{Xu} \mathrm{H}$ : Selective propagation of functional mitochondrial DNA during oogenesis restricts the transmission of a deleterious mitochondrial variant. Nat Genet 46: 389-392, 2014.

26. Viale A, Pettazzoni P, Lyssiotis CA, Ying H, Sánchez N, Marchesini M, Carugo A, Green T, Seth S, Giuliani V, et al: Oncogene ablation-resistant pancreatic cancer cells depend on mitochondrial function. Nature 514: 628-632, 2014.

27. Berridge MV, Dong L and Neuzil J: Mitochondrial DNA in Tumor Initiation, Progression, and Metastasis: Role of Horizontal mtDNA Transfer. Cancer Res 75: 3203-3208, 2015.

28. Stefano GB, Mantione KJ, Casares FM and Kream RM: Anaerobically functioning mitochondria: Evolutionary perspective on modulation of energy metabolism in Mytilus edulis. Invertebrate Surviv J 12: 22-28, 2015.

29. Stefano GB, Snyder C and Kream RM: Mitochondria, chloroplasts in animal and plant cells: Significance of conformational matching. Med Sci Monit 21: 2073-2078, 2015.

30. Warburg O, Gawehn K, Geissler AW, Kayser D and Lorenz S: Experiments on anaerobiosis of cancer cells. Klin Wochenschr 43: 289-293, 1965 (In German).

31. Seyfried TN: Cancer as a mitochondrial metabolic disease. Front Cell Dev Biol 3: 43, 2015.

32. Seyfried TN, Flores R, Poff AM, D'Agostino DP and Mukherjee P: Metabolic therapy: A new paradigm for managing malignant brain cancer. Cancer Lett 356 (2 Pt A): 289-300, 2015.

33. Stefano GB and Kream RM: Cancer: Mitochondrial origins. Med Sci Monit 21: 3736-3739, 2015.

34. Snyder C, Kream RM, Ptacek R and Stefano GB: Mitochondria, microbiome and their potential psychiatric modulation. Autism Open Access 5: 144, 2015.

35. Fujimura KE, Slusher NA, Cabana MD and Lynch SV: Role of the gut microbiota in defining human health. Expert Rev Anti Infect Ther 8: 435-454, 2010 
36. Guinane CM and Cotter PD: Role of the gut microbiota in health and chronic gastrointestinal disease: Understanding a hidden metabolic organ. Therap Adv Gastroenterol 6: 295-308, 2013.

37. Greaves LC, Reeve AK, Taylor RW and Turnbull DM: Mitochondrial DNA and disease. J Pathol 226: 274-286, 2012.

38. Li M, Schröder R, Ni S, Madea B and Stoneking M: Extensive tissue-related and allele-related mtDNA heteroplasmy suggests positive selection for somatic mutations. Proc Natl Acad Sci USA 112: 2491-2496, 2015.

39. Goto H, Dickins B, Afgan E, Paul IM, Taylor J, Makova KD and Nekrutenko A: Dynamics of mitochondrial heteroplasmy in three families investigated via a repeatable re-sequencing study. Genome Biol 12: R59, 2011.

40. Allen JF: Why chloroplasts and mitochondria retain their own genomes and genetic systems: Colocation for redox regulation of gene expression. Proc Natl Acad Sci USA 112: 10231-10238, 2015.

41. Xu X, Duan S, Yi F, Ocampo A, Liu GH and Izpisua Belmonte JC: Mitochondrial regulation in pluripotent stem cells. Cell Metab 18 : 325-332, 2013

42. Prigione A, Rohwer N, Hoffmann S, Mlody B, Drews K, Bukowiecki R, Blümlein $\mathrm{K}$, Wanker $\mathrm{EE}$, Ralser $\mathrm{M}$, Cramer T, et al: HIF1 $\alpha$ modulates cell fate reprogramming through early glycolytic shift and upregulation of PDK1-3 and PKM2. Stem Cells 32: 364-376, 2014.
43. Stefano GB and Kream RM: Hypoxia defined as a common culprit/initiation factor in mitochondrial-mediated proinflammatory processes. Med Sci Monit 21: 1478-1484, 2015.

44. Campello S, Lacalle RA, Bettella M, Mañes S, Scorrano L and Viola A: Orchestration of lymphocyte chemotaxis by mitochondrial dynamics. J Exp Med 203: 2879-2886, 2006.

45. Campello S and Scorrano L: Mitochondrial shape changes: Orchestrating cell pathophysiology. EMBO Rep 11: 678-684, 2010.

46. Wallace DC: A mitochondrial paradigm of metabolic and degenerative diseases, aging, and cancer: A dawn for evolutionary medicine. Annu Rev Genet 39: 359-407, 2005.

47. Calloway CD, Reynolds RL, Herrin GL Jr and Anderson WW: The frequency of heteroplasmy in the HVII region of mtDNA differs across tissue types and increases with age. Am J Hum Genet 66: 1384-1397, 2000.

48. Snyder C and Stefano GB: Mitochondria and chloroplasts shared in animal and plant tissues: Significance of communication. Med Sci Monit 21: 1507-1511, 2015. 